\title{
Formulação e caracterização de potenciais filamentos compósitos de PLA e talos de tabaco para aplicação em manufatura aditiva
}

\author{
Formulation and characterization of potential \\ composites filaments from PLA and tobacco stems \\ for application in additive manufacturing
}

\author{
Fernanda Rech ${ }^{1}$, Sara Magalhães da Silva ${ }^{2}$, \\ Liane Roldo $^{3}$, José Martinho Oliveira ${ }^{2}$, Fabio Pinto Silva ${ }^{1}$
}

\footnotetext{
${ }^{1}$ Universidade Federal do Rio Grande do Sul (UFRGS), Programa de Pós-Graduação em Design (PGDESIGN/UFRGS), Av. Osvaldo Aranha, 99 , $6^{\circ}$ andar (sala 607), CEP: 90035-190, Porto Alegre, Rio Grande do Sul, Brasil.

${ }^{2}$ Universidade de Aveiro (UA), Escola Superior de Design, Gestão e Tecnologia de Produção Aveiro-Norte (ESAN), Estrada do Cercal, 449, CEP: 3720-509, Oliveira de Azeméis, Portugal.

${ }^{3}$ University of Split, Faculty of Maritime Studies, Rudera Boškovića 37, HR 21000, Split, Croatia.

e-mail: fernandarechmoda@gmail.com, lroldo@pfst.hr, fabio.silva@ufrgs.br, sarapms@ua.pt, martinho@ua.pt
}

\section{RESUMO}

A tecnologia de fabricação por filamento fundido (FFF) utilizando polímeros como PLA e ABS é bem conhecida. Contudo, o desenvolvimento de filamentos compósitos para esta tecnologia de manufatura aditiva, bem como a determinação dos parâmetros de processamento e das propriedades resultantes na extrusão e na impressão 3D, ainda requerem pesquisa. Esse estudo exploratório analisa as propriedades químicas, térmicas e a morfologia de filamento de matriz polimérica de PLA e talos de tabaco, visando sua utilização no processo FFF. Os materiais utilizados foram talos de tabaco moídos e PLA. As partículas de tabaco, com tamanho médio de $50 \mu \mathrm{m}$, apresentaram-se aleatoriamente distribuídas no filamento. No entanto, aglomerados de partículas, bolhas e porosidades foram observados no centro do filamento. Ensaios por espectroscopia no infravermelho por transformada de Fourier mostraram que o filamento compósito apresentou interação mecânica entre os materiais. A análise térmica permitiu verificar que não houve degradação do pó de talos de tabaco, mantendo a integridade química e física do filamento compósito a temperatura de extrusão de $180{ }^{\circ} \mathrm{C}$. A calorimetria exploratória diferencial revelou que as partículas de pó de talos de tabaco podem ter atuado como agente nucleante, aumentando o grau de cristalinidade do filamento compósito. Sugere-se para estudos futuros o aumento do percentual de pó de talos de tabaco, a investigação de outros métodos de preparação de compósitos, bem como um estudo da adição de plastificantes ou agentes compatibilizantes para aprimorar a ligação entre a interface do PLA e as partículas de tabaco.

Palavras-chave: impressão 3D, fabricação de filamento fundido, manufatura aditiva, compósitos, tabaco.

\section{ABSTRACT}

Fused filament fabrication (FFF) using polymers such as PLA and ABS are well known. However, the development of composite filaments for this type of additive manufacturing technology, as well as the study of processing parameters and properties resulting from extrusion process and printing still require investigation. This exploratory research aims to analyze chemical and thermal properties and the morphology of a composite filament from PLA polymer matrix and tobacco stems to seek its use in the FFF process. The materials used were waste of tobacco stems powder and PLA. Tobacco particles, with an average size of $50 \mu \mathrm{m}$, have distribution on the filament. However, clusters of particles, bubbles and porosities were observed in the center of the filament. Fourier Transform Infrared analyses showed that the composite filament presented mechanical interaction between the materials. Thermal analyses allowed to verify that there was no degradation of tobacco stem powder. Therefore, chemical and physical integrity of the composite filament was kept at an extrusion temperature of $180{ }^{\circ} \mathrm{C}$. Differential scanning calorimetry revealed that micronized particles of tobacco stems may have acted as nucleating agents for crystallization, increasing the crystallinity degree of the 
composite filament. It is recommended for future studies to increase the percentage of tobacco stems powder, the investigation of other methods for composites preparation, as well as a study of the addition of plasticizers or coupling agents to improve the interface between PLA and tobacco particles.

Keywords: 3D printing, fused filament fabrication, additive manufacturing, composites, tobacco.

\section{INTRODUÇÃO}

Processos de fabricação por manufatura aditiva (AM) ${ }^{1}$, usando materiais de origem biológica [1, 2], tem sido continuamente incentivados devido a questões ambientais, econômicas, pelo aprimoramento da tecnologia e dos materiais compósitos de matrizes vegetais e poliméricas de origem renovável, os chamados biocompósitos [3]. Esses materiais vegetais utilizados consistem principalmente em resíduos provenientes da indústria ou da agricultura, e são, em sua maioria produzidos localmente [3].

A fabricação por filamento fundido é o processo AM mais difundido, principalmente para uso doméstico [3-5]. Essa tecnologia apresenta baixo custo [3, 6] e é mais simples de manusear em comparação a outros processos aditivos [4].

FFF consiste em construir partes camada por camada, aquecendo um filamento termoplástico até um estado fundido e viscoso. O polímero fundido é extrudado através de um pequeno bico extrusor, e, em seguida, depositado em camadas sobre uma plataforma [3, 5, 7-10]. Cada camada depositada segue um padrão calculado pelo software de controle da impressora, que reproduz a geometria desejada do objeto, inserida através de um arquivo $\mathrm{CAD}^{3}$, normalmente no formato STL [3]. Uma vez depositado e coberto pela camada seguinte, formam-se sobreposições que podem criar rugosidade, dependendo do material, da proporção da peça, do bico de extrusão e da qualidade de fundição [5].

Entre as vantagens do FFF estão: a liberdade de geometrias, que permite a construção de projetos com formas complexas e distintas, a diminuição do número de montagens e de desperdício [10], a redução do tempo de espera e da necessidade de armazenamento e transporte, uma vez que permite a produção de peças simultaneamente, possibilitando a produção de um produto completo. A manufatura tradicional geralmente inclui a produção em vários locais, o que torna necessário maior capacidade de armazenamento [11]. Entre as dificuldades cita-se a existência de diversos parâmetros de processamento que estão diretamente relacionados com o design da peça final, contribuindo para a qualidade e funcionalidade da mesma $[4,7,9]$. Entre alguns parâmetros podem-se citar: direção de construção, temperatura de extrusão, espessura da camada, padrão de preenchimento e velocidade de impressão [7].

O poli(ácido lático) - PLA é um polímero termoplástico comumente utilizado para a fabricação de filamentos para o processo de impressão por FFF. O PLA é um poliéster alifático, biodegradável e de origem renovável. Em relação a sua estrutura, pode ser amorfo ou semi cristalino. Pelo fato de possuir boas propriedades de coalescência, é um polímero adequado para FFF. Apesar de ter um índice de fluidez (MFI) moderado, (entre 3 a 15 g/10 min), possui uma baixa viscosidade de fusão. Por essa razão, apresenta baixa pressão de processamento. Ainda, quando submetido a um pequeno aumento de temperatura, a sua viscosidade diminui, tornando esse polímero ideal para FFF. Em relação às propriedades mecânicas, possui alta resistência e rigidez, embora seja quebradiço $[12,13]$.

A relação do PLA com a tecnologia FFF ocorre principalmente no campo biomédico, em particular na produção de engenharia de tecidos, pois esse material favorece a adesão e proliferação celular, proporcionando um ambiente positivo do ponto de vista biológico e mecânico. Entre as vantagens do PLA estão a biocompatibilidade $[14,15]$, a processabilidade $[14,16]$ e melhor qualidade de impressão devido à redução do encolhimento [16]. O fato de ser quimicamente inerte, a baixa tenacidade, a baixa taxa de degradação e a hidrofobicidade são algumas de suas desvantagens [14].

A vantagem da aplicação de polímeros preenchidos com materiais naturais para reforçar filamentos ter-

\footnotetext{
${ }^{1}$ Manufatura Aditiva (AM), geralmente conhecida como impressão 3D, é o termo geral para tecnologias que utilizam a deposição de sucessivas camadas para desenvolver objetos físicos a partir de uma representação geométrica. A utilização dessas tecnologias aplica-se em um diversificado número de áreas como engenharia, medicina, educação, arquitetura, cartografia, indústria de brinquedos e entretenimento [50].

${ }^{2} \mathrm{O}$ processo por FFF é também comumente designado por FDM (Modelagem por Fusão e Deposição). Os processos envolvidos na impressão por FFF e FDM são os mesmos. A diferença entre ambos é que o termo FDM é uma marca comercial, registrada pela Stratasys Inc. em 1989

${ }^{3}$ Design Assistido por computador (Computer-Aided Design).
} 
moplásticos para FFF está baseada na redução de custos, aumento da resistência à flexão e melhoria na estabilidade dimensional após a solidificação, além da redução do impacto ambiental [3]. WIJK e WIJK [1] destacam o surgimento da produção de filamentos poliméricos a partir de recursos renováveis como a biomassa, e sinalizam o aparecimento de uma diversidade no que tange o desenvolvimento de novos produtos.

Materiais lignocelulósicos, como as fibras vegetais, possuem propriedades específicas, como baixa densidade. Além disso, apresentam alta resistência elétrica e propriedades de isolamento acústico [17-20]. São biodegradáveis e devido a baixa densidade, seu processamento consome menos energia [21]. Ainda, seu uso possibilita fomentar a geração de trabalho e renda na zona rural [22].

O uso de filamentos biocompósitos termoplásticos para a tecnologia FFF é recente e tem aparecido na literatura principalmente a partir do ano de 2016. Um interesse crescente da comunidade científica e a disponibilidade de filamentos comerciais confirmam o interesse do mercado. Alguns desafios no processamento ainda são recorrentes como a falta de homogeneidade na dispersão do material vegetal e a obtenção de vazios durante o processamento. Faz-se necessário criar uma boa interface entre o polímero e a fibra natural [3].

A hidrofilicidade dos materiais de origem vegetal e a hidrofobicidade do PLA, pode traduzir-se em uma fraca ligação entre os materiais, prejudicando a interface dos mesmos. Uma eficiente adesão entre a fase dispersa e a matriz, produz uma interface resistente a esforços mecânicos, por sua vez, um compósito com melhores propriedades e desempenhos [18].

A respeito do tabaco, a relevância da fumicultura no território brasileiro é notória. O Brasil é o segundo maior produtor mundial de tabaco e líder em exportações desde 1993 [23]. A maior parte da produção nacional está localizada na região Sul, que é responsável por 97\% de toda a produção do país [24].

Em relação à planta do tabaco (Nicotiana tabacum L.), sua produção está voltada para o fornecimento de folhas de alta qualidade para a fabricação de cigarros. O principal resíduo da fumicultura é o caule, e, em algumas espécies de tabaco, como fumo de corda, os talos também são inutilizados após a colheita.

A geração anual mundial do caule do tabaco é estimada em 3,2 milhões de toneladas, o que representa $40 \%$ da produção total mundial [25]. QIN et al. [26] afirmam que o final do ciclo de vida desse resíduo agrícola é geralmente inadequado, que acaba sendo abandonado nas plantações ou queimado a céu aberto. Além disso, a presença de nicotina nos caules provoca um aumento na eliminação de resíduos sólidos e problemas de poluição ambiental [27].

O resíduo do caule do tabaco é uma biomassa fibrosa formada por celulose, hemicelulose e lignina. Seus componentes químicos básicos se assemelham as espécies de madeira folhosas de folha larga, mas com menor densidade [27-29].

Neste trabalho, a escolha pela utilização do resíduo agrícola da planta do tabaco foi tomada não apenas levando-se em consideração o significativo volume gerado pela fumicultura brasileira, mas também enfatizando a preocupação ambiental. Além da preocupação ambiental, evidencia-se a dimensão social da investigação, tendo em vista que propõe nova utilização para o rejeito agrícola em setor distinto da indústria fumageira, promovendo a diversificação da atividade produtiva dos fumicultores. Serão utilizados resíduos provenientes da produção de fumo de corda.

O fumo de corda, também conhecido como fumo de rolo, é produzido em localidades do sul do Brasil e consiste em um processo de produção artesanal, afazer aprendido de pai para filho e desassociado do mercado transnacional da indústria fumageira, atividade que iniciou na década de 60 [30]. Durante sua produção, o talo é descartado. Por esta razão, ele é o resíduo agrícola escolhido para essa investigação.

Tendo em vista o contexto descrito, o presente artigo objetiva desenvolver e caracterizar química, térmica e morfologicamente um filamento compósito de talos de tabaco e PLA, visando verificar seu potencial de utilização para posterior aplicação no processo de fabricação FFF.

\section{MATERIAIS E MÉTODOS}

\subsection{Materiais}

As matérias-primas utilizadas foram grânulos virgens de PLA 9001 da marca Corbion, fornecidos pela empresa 2M3D (Novo Hamburgo, RS, Brasil) e talos de tabaco moído do tipo corda, comumente conhecido como fumo de rolo, proveniente da cidade de Segredo, localizada no Vale do Rio Pardo ${ }^{4}$.

\footnotetext{
${ }^{4} \mathrm{O}$ Vale do Rio Pardo está localizado na região sul do Brasil e é formado pelos seguintes municípios: Arroio do Tigre, Boqueirão do Leão, Candelária, Encruzilhada do Sul, Estrela Velha, General Câmara, Herveiras, Ibarama, Lagoa Bonita do Sul, Mato Leitão, Pantano
} 


\subsection{Extrusão de Filamento}

Os filamentos compósitos de talos de tabaco e PLA virgem foram elaborados a partir do processo de extrusão, em extrusora do tipo monorrosca da marca SEIBT, modelo ES 35 a uma temperatura média de $180{ }^{\circ} \mathrm{C}$. A temperatura de cada zona da extrusora pode ser visualizada na tabela 1. A velocidade de extrusão (motor da extrusora) foi de $663,6 \mathrm{rpm}$. Os filamentos apresentaram um diâmetro médio de $0,85 \mathrm{~mm}$, com um desvio padrão de $0,8 \mathrm{~mm}$.

Tabela 1: Temperaturas de extrusão.

\begin{tabular}{c|c|c|c|c}
\hline ZONA 1 & ZONA 2 & ZONA 3 & ZONA 4 & ZONA 5 \\
\hline $179^{\circ} \mathrm{C}$ & $183^{\circ} \mathrm{C}$ & $183^{\circ} \mathrm{C}$ & $179^{\circ} \mathrm{C}$ & $176^{\circ} \mathrm{C}$ \\
\hline
\end{tabular}

Os talos secos foram inicialmente processados em um moinho de facas da marca SEIBT, modelo MGHS $1.5 / 85$, motor com potência de $2 \mathrm{hp}$ e peneira de $6 \mathrm{~mm}$ de diâmetro, e posteriormente micronizados em micronizador AX PLASTICOS Modelo 001 com uma velocidade de rotação de $1700 \mathrm{rpm}$.

O material micronizado foi então peneirado, sendo retiradas e eliminadas as partículas maiores que 14 mesh $(1,41 \mathrm{~mm})$. O processo de moagem do material restante foi realizado em um moinho planetário de bolas (CT - 242), da marca Servitech durante 15 minutos. Após a finalização da moagem, uma amostra do material foi encaminhada para realização de ensaio de granulometria a laser, com o intuito de verificar o tamanho das partículas obtidas.

A mistura do pó de talos de tabaco com o PLA foi realizada de forma manual, levando em consideração percentagens pré-estabelecidas. A proporção utilizada das matérias-primas foi de $1 \% \mathrm{~m} / \mathrm{m}$ de pó de talos de tabaco e $99 \% \mathrm{~m} / \mathrm{m}$ de PLA, conforme a Tabela 2.

Tabela 2: Materiais utilizados para o processo de extrusão.

\begin{tabular}{c|c|c}
\hline DESCRIÇÃO & MATERIAL & PROPORÇÃO (\%) \\
\hline Filamento PLA virgem & Pellets virgem de PLA & 100 \\
\hline Filamento compósito & Pellets virgem de PLA & 99 \\
& Pó de talo de tabaco tipo corda. & 1 \\
\hline
\end{tabular}

\subsection{Caracterizações}

O ensaio de granulometria a laser foi realizado à líquido (água) no equipamento Cilas 1180 . Não foi feito uso de agente dispersante. O ensaio teve como intuito verificar o tamanho das partículas do material vegetal. Essa etapa antecedeu o processo de extrusão.

Microscopia eletrônica de varredura (MEV) e microscopia óptica (MO) foram utilizadas para verificar características morfológicas. Com auxílio de uma lâmina com anteparo, foram cortados finos filetes no sentido longitudinal de aproximadamente $7 \mathrm{~mm}$ de comprimento. As amostras preparadas foram observadas em microscópio óptico da marca Nikon (Eclipse L150). As imagens foram capturadas usando uma câmera digital Canon EOS 100D com resolução de 5184 x 3456 pixels. As análises por MEV foram realizadas no equipamento da marca Hitachi e modelo TM3000-Tabletop Microscope. Foram elaborados, em diferentes zonas do filamento, cortes no sentido transversal, utilizando uma lâmina com anteparo. As amostras foram analisadas sem recobrimento, uma vez que o equipamento utilizado é de baixo vácuo.

A composição química foi determinada por espectroscopia no infravermelho por transformada de Fourier (FTIR), modo ATR, em equipamento modelo IRAffinity-1, da Shimadzu. A resolução utilizada foi de $4 \mathrm{~cm}^{-}$ ${ }^{1}$, com 16 varreduras, por amostra, na região de absorção de 4000 a $600 \mathrm{~cm}^{-1}$. O FTIR foi realizado em três amostras distintas: no talo de tabaco, no filamento compósito de talos de tabaco moído e PLA, e no filamento apenas com PLA.

As propriedades térmicas do filamento compósito, do filamento de PLA virgem e do pó de talos de fumo de corda foram determinadas por calorimetria exploratória diferencial (DSC) e análise termogravimétrica (TGA). O equipamento Netzsch, modelo DSC 404 F1 Pegasus, com uma taxa de aquecimento de $10^{\circ} \mathrm{C} / \mathrm{min}$

Grande, Passa Sete, Passo do Sobrado, Rio Pardo, Santa Cruz do Sul, Segredo, Sinimbu, Sobradinho, Tunas, Vale do Sol, Vale Verde, Venâncio Aires e Vera Cruz. Informações retiradas do site: Disponível em: <http://www.fee.rs.gov.br/perfilsocioeconomico/coredes/detalhe/?corede=Vale+do+Rio+Pardo>. Acesso em: 22 out. 2019. 
em atmosfera de nitrogênio foi utilizado para o DSC. Entalpia de fusão $\left(\Delta \mathrm{H}_{\mathrm{m}}\right)$ e de cristalização à frio $\left(\Delta \mathrm{H}_{\mathrm{cc}}\right)$ foram calculadas e o grau de cristalinidade foi determinado pela Equação 1, de acordo com a investigação publicada por SILVA et al [31], tendo em vista a diferença que no presente estudo $w$ é o peso percentual do pó de talos de tabaco.

$$
x_{c}=\frac{\Delta H_{m}-\Delta H_{c e}}{\Delta H_{m}^{0}(1-w)} \times 100
$$

A análise termogravimétrica (TGA) do pó de talos de fumo de corda foi realizada no equipamento $50 \mathrm{H}$ da Shimadzu a uma faixa de temperatura de $30{ }^{\circ} \mathrm{C}$ a $800{ }^{\circ} \mathrm{C}$, com taxa de aquecimento de $10{ }^{\circ} \mathrm{C} / \mathrm{min}$ em atmosfera de nitrogênio.

\section{RESULTADOS E DISCUSSÃO}

O ensaio de granulometria (Figura 1) mostra que o diâmetro médio das partículas foi de $50 \mu \mathrm{m}$. O diâmetro a $90 \%$ foi de $102 \mu \mathrm{m}$, a $50 \%$ foi de $27 \mu \mathrm{m}$ e o diâmetro a $10 \%$ foi de $5,25 \mu \mathrm{m}$. Em investigação semelhante, porém com resíduo vegetal distinto, TAO et al. [32] sugerem um tamanho médio de partícula de $14 \mu \mathrm{m}$ para farinha de madeira, obtida a partir de serragem de madeira seca. Já WAHAB et al. [33] utilizaram resíduos agrícolas com base na madeira com aproximadamente $90 \mu \mathrm{m}$. ANTUNES [34] utilizou partículas de pó de cortiça menores que $40 \mu \mathrm{m}$ e TRAN et al. [35], ao desenvolverem filamento a partir de resíduos da casca do cacau e PCL (Policaprolactona), utilizaram partículas menores que $63 \mu \mathrm{m}$. Tendo em vista a dimensão das partículas utilizadas em investigações semelhantes, considera-se que o tamanho de partícula obtido após a etapa de moagem é suficiente para a presente investigação.

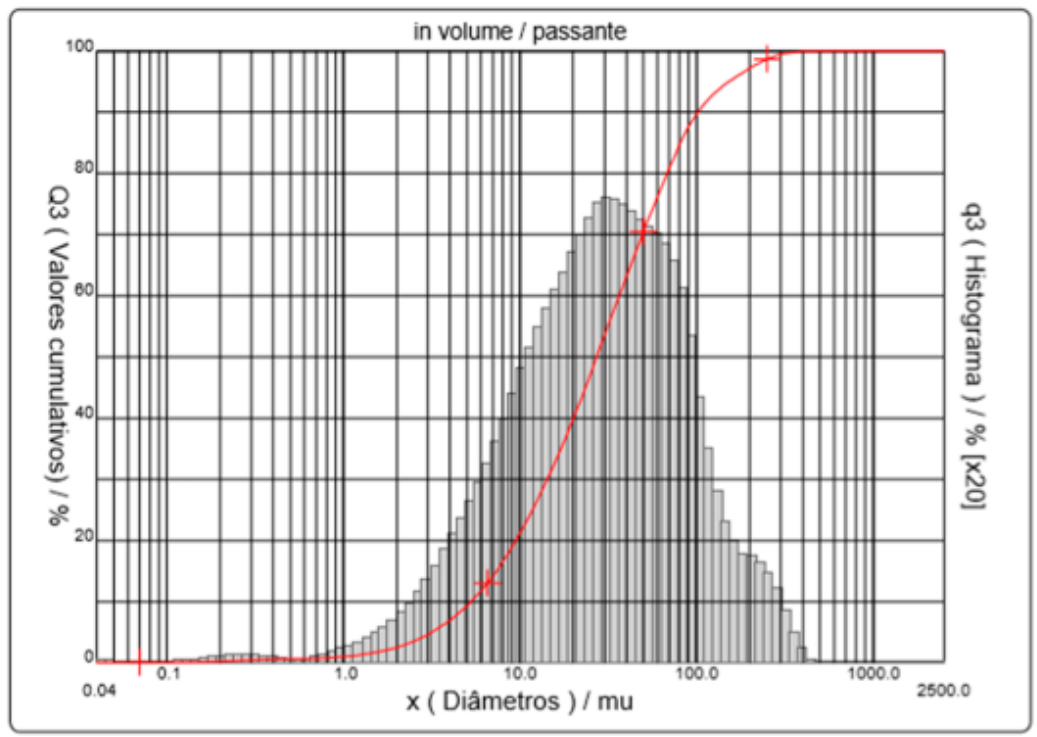

Figura 1: Histograma mostrando a curva de distribuição do tamanho de partícula em porcentagem e em volume.

Pode-se verificar na Figura 2A o filamento compósito desenvolvido a partir de PLA virgem e pó de talos de tabaco do tipo corda. As partículas de tabaco, que, de acordo com a Figura 1 encontram-se em sua maioria entre 5 e $200 \mu \mathrm{m}$, estão dispersas ao longo do filamento compósito - Figura 2B. 

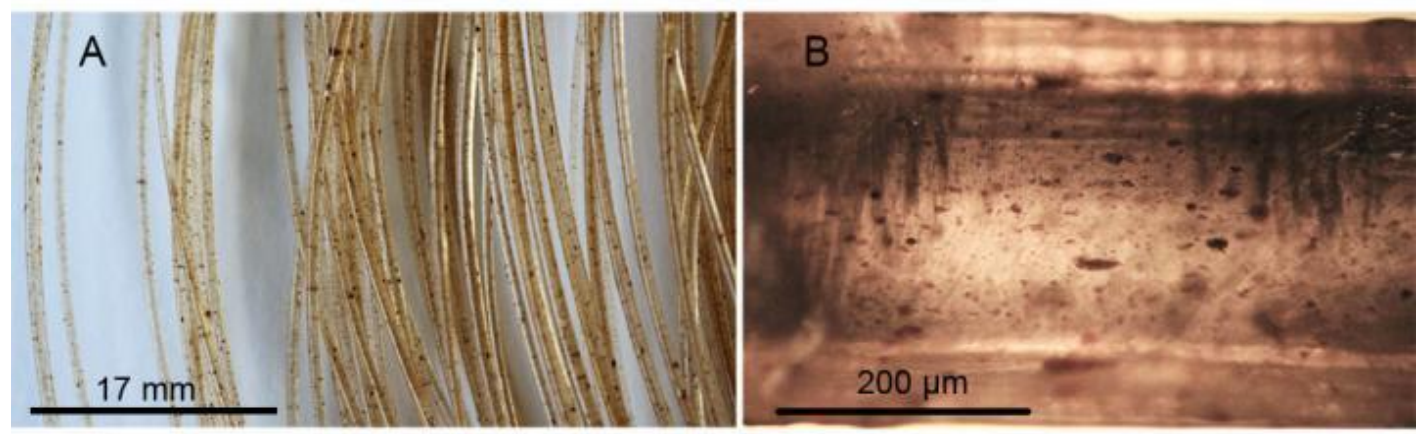

Figura 2: (a) filamento compósito extrudado (b) imagem de microscopia óptica do corte longitudinal do filamento compósito.

Em relação à análise de MEV, na micrografia apresentada na Figura 3a, percebe-se que as partículas do PLA e as partículas de pó de tabaco e cargas, provenientes do PLA, estão aleatoriamente distribuídas no filamento compósito. Contudo, na região central do filamento, constatou-se aglomeração de partículas, bolhas e porosidades. Observa-se na Figura 3b, a distribuição de cargas no PLA virgem.

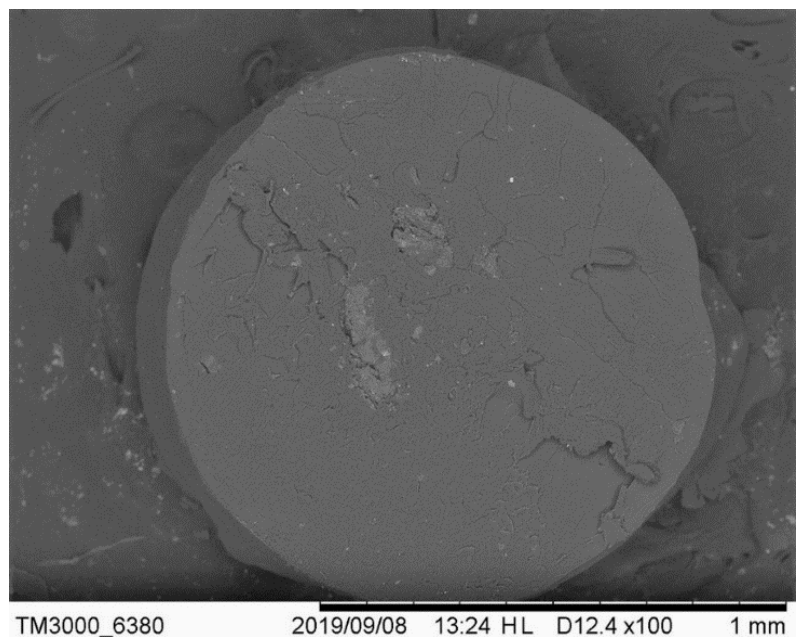

(a)

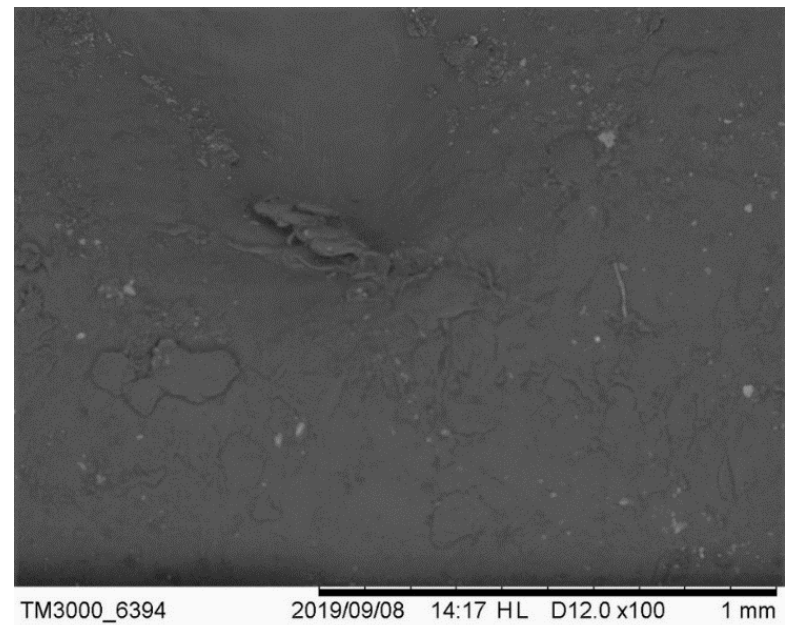

(b)

Figura 3: Imagem de MEV do corte transversal em (a) amostra do filamento compósito de talos de tabaco moído e PLA e (b) amostra do filamento de PLA virgem evidenciando a presença de cargas.

Espaços vazios podem ser observados entre as interfaces do pó e do polímero - Figura 4a e 4b. De acor- 
do com TAO et al. [32], isso pode indicar uma ineficiência na ligação interfacial entre os materiais, possivelmente devido ao pó dos talos de tabaco apresentar uma superfície polar (hidrofílica) e o PLA uma superfície não polar (hidrofóbica). Sendo assim, em teoria, a força da interface entre ambos os materiais é baixa. Entretanto, a força da interface pode ser aumentada melhorando a compatibilidade do material [32] por meio da introdução de agentes compatibilizantes. Agentes compatibilizantes são compostos químicos que possibilitam aumentar e melhorar a adesão interfacial entre materiais de naturezas distintas, uma vez que reduzem tensões interfaciais entre a fase dispersa e a matriz, apresentando um efeito emulsificante [34, 36-38]. Atuam, promovendo a união entre duas partes, resultando em uma mistura com interações químicas e não somente mecânicas.

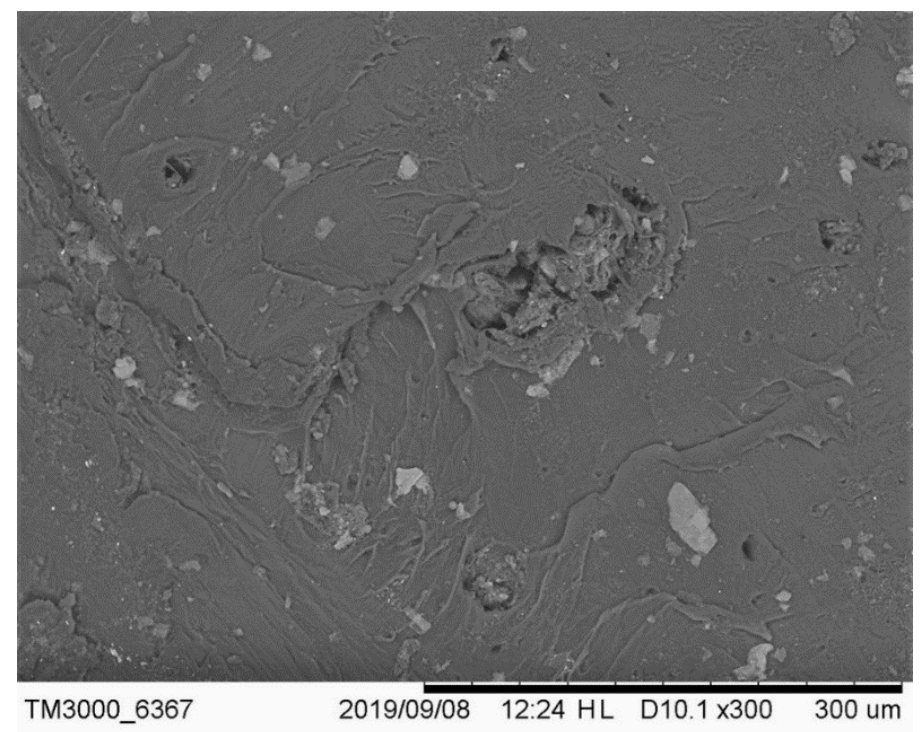

(a)

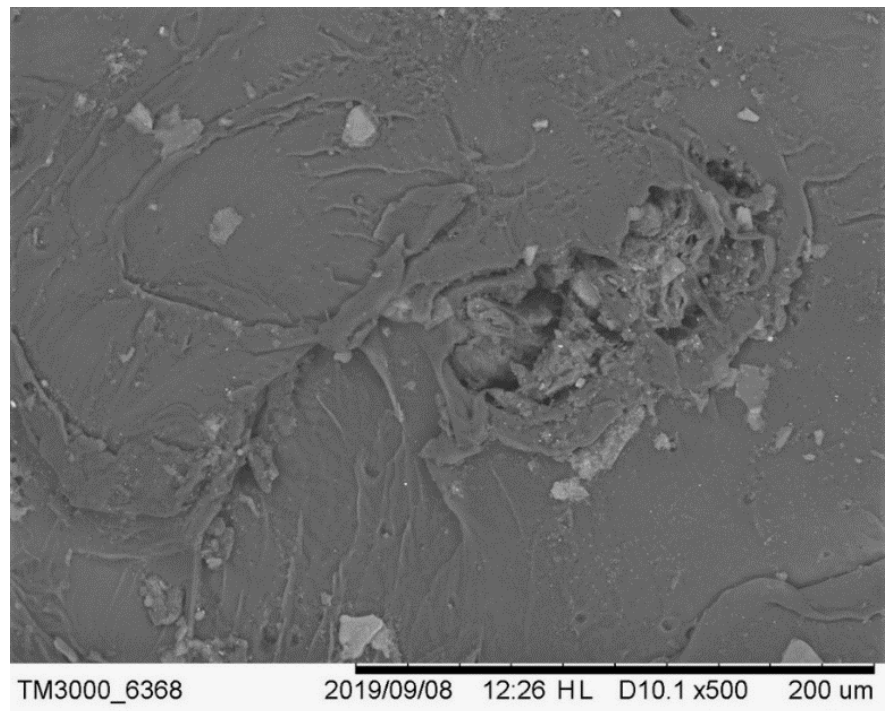

(b)

Figura 4: (a) detalhe da distribuição das partículas e (b) aglomerado de partículas no centro do filamento, evidência de interface entre o material vegetal e o polímero.

Em relação ao FTIR, o espectro do talo de tabaco (Figura 5a) apresenta uma banda de vibração a 1732 $\mathrm{cm}^{-1}$, indicando a presença do grupo de carbonila $(\mathrm{C}=\mathrm{O})$. Esse grupo dá origem a uma forte absorção na região de 1880 a $1650 \mathrm{~cm}^{-1}$. As bandas a $3340 \mathrm{~cm}^{-1}$ e $2918 \mathrm{~cm}^{-1}$ possivelmente representam compostos de $\mathrm{OH}$, que incluem água absorvida, álcoois alifáticos primários e secundários, provavelmente identificados em celulose, hemicelulose, lignina e ácidos carboxílicos. A banda a $1238 \mathrm{~cm}^{-1}$ sinaliza a presença de estiramento de C-O. Ligações duplas ou anéis aromáticos podem aparecer na banda a $1639 \mathrm{~cm}^{-1}$, uma vez que C=C dá 
origem a uma banda fraca próxima de $1650 \mathrm{~cm}^{-1}$. Absorções de intensidade média a forte na região de 1600 a $1450 \mathrm{~cm}^{-1}$, estão relacionadas a vibrações de anel aromático. A banda a $1504 \mathrm{~cm}^{-1}$ provavelmente caracteriza a existência de anéis aromáticos $(\mathrm{C}=\mathrm{C})$, já a banda $1372 \mathrm{~cm}^{-1}$ pode estar relacionada ao grupo de alcanos (pico de dobramento de $\mathrm{CH}_{3} \mathrm{em}$ aproximadamente $1375 \mathrm{~cm}^{-1}$ ). Por fim, a banda a $896 \mathrm{~cm}^{-1}$ pode estar ligada com a vibração C-H da lignina $[39,40]$.

A análise de FTIR do filamento contendo apenas PLA resultou em um espectro característico desse material (Figura 5b). A banda a $2923 \mathrm{~cm}^{-1}$ sinaliza um estiramento da ligação C-H, característico do grupo alcano [39]. Para CHIENG et al. [41], o PLA mostra frequências de estiramento características de $\mathrm{C}=\mathrm{O}$ em 1746 $\mathrm{cm}^{-1}, \mathrm{CH}_{3}$ simétrico em $2923 \mathrm{~cm}^{-1}$. A banda a $1452 \mathrm{~cm}^{-1}$ demonstra, possivelmente, a presença de deformação em C-H [41]. Conforme MOFOKENG et al. [42], as regiões de interesse para o PLA e os compósitos são a de 1780 a $1680 \mathrm{~cm}^{-1}$, para o estiramento de $\mathrm{C}=\mathrm{O}$, e a de 3600 a $3000 \mathrm{~cm}^{-1}$, para o estiramento O-H. Esta última banda não é visível no espectro. No entanto, as bandas em aproximadamente $1750 \mathrm{e} 1180 \mathrm{~cm}^{-1}$, que pertencem ao estiramento $\mathrm{C}=\mathrm{O}$ e estiramento $\mathrm{C}-\mathrm{O}-\mathrm{C}$, estão visíveis no espectro.

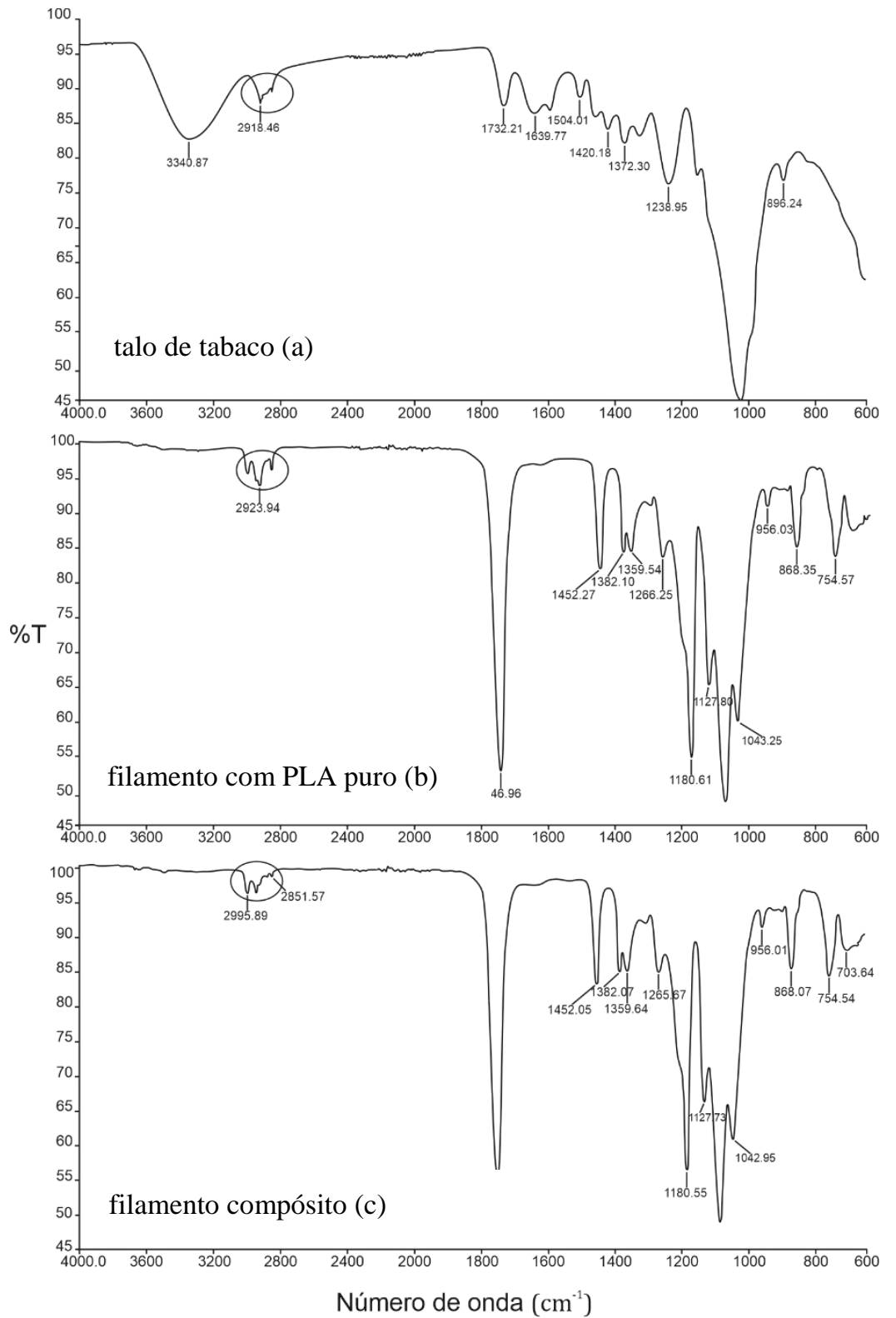

Figura 5: Espectros de FTIR do (a) pó de talo de tabaco, (b) filamento com PLA puro e (c) filamento compósito.

Pode ser observado na Figura 5c - espectro do filamento compósito, que a inserção de material vegetal alterou a configuração do espectro nas bandas entre 2995 e $2851 \mathrm{~cm}^{-1}$. Entre outros fenômenos, como o da cristalização do PLA, é possível perceber que houve uma redução da intensidade de algumas bandas referen- 
tes ao pó de talos de tabaco, possivelmente devido a sua baixa carga. Além disso, não pode-se admitir que houve quaquer interação química entre os materiais (PLA e pó de talos de tabaco). A análise dos espectros do FTIR indica a presença de duas fases, resultantes da interação mecânica da mistura. Essa análise permite complementar e validar os resultados obtidos nas imagens de MEV, que mostram falta de adesão interfacial entre os materiais.

MOFOKENG et al. [42] ao analisar o espectro de um compósito de PLA com fibra de sisal, observaram o surgimento de uma pequena banda, logo abaixo do pico de carbonila, a $1650 \mathrm{~cm}^{-1}$. Essa banda é, possivelmente, proveniente do composto $\mathrm{O}-\mathrm{H}$ que se originou da deformação do grupo hidroxila da água absorvida durante a extrusão do compósito, normalmente acumulada na celulose [37]. Conforme pode ser observado na Figura 5c, esse pico não aparece no espectro. Uma possível razão poderá ser a pequena porcentagem de talos de tabaco utilizada para o desenvolvimento do compósito.

Na Figura 6 encontram-se representadas as curvas de DSC dos materiais ensaiados. Através desta Figura verifica-se que ambos os materiais degradam-se a temperaturas superiores a $250{ }^{\circ} \mathrm{C}$, onde a degradação para o filamento apenas com PLA tem início a uma temperatura ligeiramente superior $\left(\sim 280{ }^{\circ} \mathrm{C}\right)$. $\mathrm{O}$ filamento do PLA virgem apresenta um perfil de um material amorfo. A introdução de pó de talos de tabaco conduziu à alteração do perfil térmico do PLA.

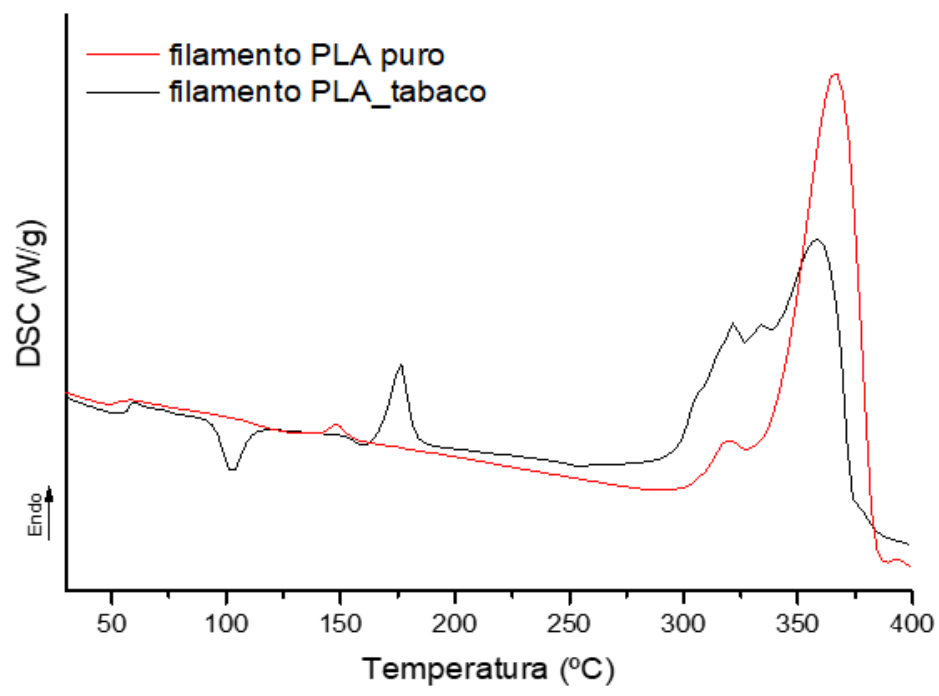

Figura 6: DSC do filamento compósito e do filamento apenas com PLA.

Para o filamento compósito é visível o aparecimento de uma $\mathrm{T}_{\mathrm{cc}}$ e o surgimento de um pico de $\mathrm{T}_{\mathrm{m}}$ mais intenso e afunilado, indicando uma alteração na cristalinidade do PLA. Os parâmetros térmicos determinados para ambos os filamentos podem ser consultados na Tabela 3.

Tabela 3: Parâmetros térmicos do filamento compósito e do filamento apenas com PLA.

\begin{tabular}{l|c|c|c|c|c|c|c|c}
\hline AMOSTRA & $\mathbf{T}_{\mathbf{g}}\left({ }^{\circ} \mathrm{C}\right)$ & $\mathbf{T}_{\mathrm{cc}}\left({ }^{\circ} \mathrm{C}\right)$ & $\Delta \mathbf{H}_{\mathrm{cc}}(\mathrm{J} / \mathrm{g})$ & $\mathbf{X}_{\mathrm{cc}}(\%)$ & $\mathbf{T}_{\mathrm{m}}\left({ }^{\circ} \mathrm{C}\right)$ & $\Delta \mathbf{H}_{\mathbf{m}}(\mathrm{J} / \mathrm{g})$ & $\mathbf{X}_{\mathbf{m}}(\%)$ & $\mathbf{X}_{\mathbf{c}}(\%)$ \\
\hline Filamento PLA virgem & 5,1 & - & - & - & 149,6 & 3,0 & 3,3 & 3,3 \\
\hline Filamento compósito & 51,5 & 101,5 & 21,8 & 23,4 & 176,5 & 26,9 & 29,0 & 5,6 \\
\hline
\end{tabular}

Os valores da $\mathrm{T}_{\mathrm{g}}$ de ambos os materiais não sofreram uma alteração significativa e estão adequados com a literatura, que descreve a $\mathrm{T}_{\mathrm{g}}$ do PLA em uma faixa de 50 a $80^{\circ} \mathrm{C}[12,43]$. No caso da $\mathrm{T}_{\mathrm{m}}$, é observável um aumento de cerca de $30^{\circ} \mathrm{C}$ para o filamento compósito. Os valores obtidos para a $\mathrm{T}_{\mathrm{m}}$ também estão de acordo com os valores reportados na literatura $130<\mathrm{T}_{\mathrm{m}}<180{ }^{\circ} \mathrm{C}[12,43]$. Este aumento da $\mathrm{T}_{\mathrm{m}}$ pode estar relacionado com o aumento do grau de cristalinidade $\left(\mathrm{X}_{\mathrm{c}}\right)$ verificado para o filamento compósito. A formação de cristais durante a cristalização do PLA na presença de tabaco conduziu a um aumento da temperatura necessária para fundir estes mesmos cristais. Esta tendência possivelmente indica uma cristalização inicial promovida pelo pó de talos de tabaco, que atuou como agente nucleante na matriz de PLA [31, 35].

O TGA (Figura 7), do pó de talos de tabaco, mostra perda de massa entre 40 e $200{ }^{\circ} \mathrm{C}$, referente à perda 
de substâncias voláteis, principlamente umidade da amostra. A maior perda de massa ocorreu entre 300 e $600{ }^{\circ} \mathrm{C}$. Nessa faixa de temperatura, os constituintes químicos estruturais das fibras vegetais estão em processo de degradação térmica. Perdas de massa a temperaturas acima de $350{ }^{\circ} \mathrm{C}$ estão associadas à degradação térmica da lignina [44].

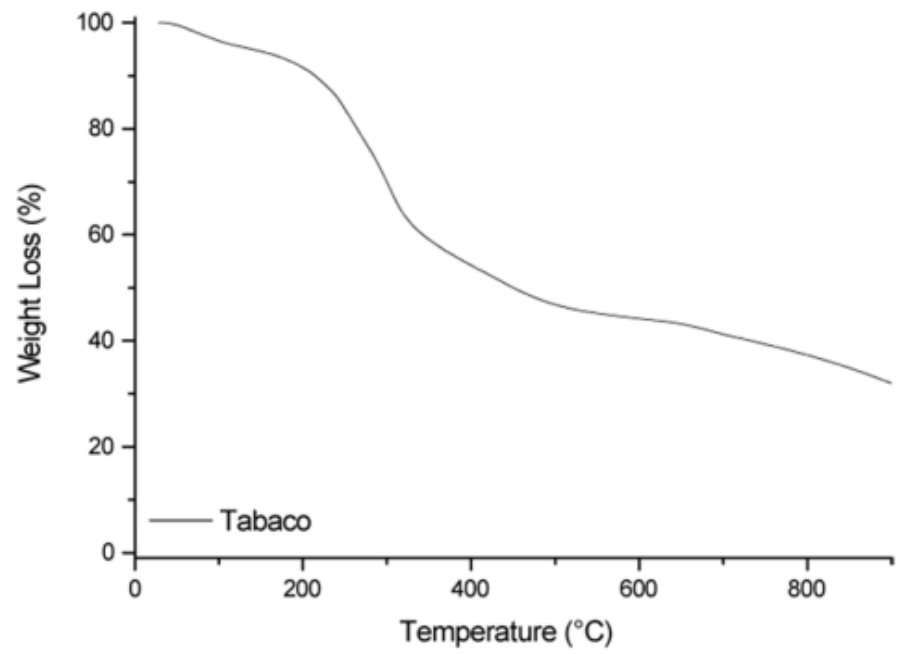

Figura 7: TGA do pó de talo de tabaco.

FONSECA [44], em estudo com a fibra da Jacitara, sugere que, com o intuito de manter a integridade química e física, as fibras devem ser submetidas a temperaturas nas quais o material tenha uma perda máxima de $40 \%$ de sua massa inicial. Tomando como base esse estudo, no caso do pó de talos de tabaco, esse material não deve ser submetido a temperaturas ultrapassem $350{ }^{\circ} \mathrm{C}$, visto que, a essa temperatura, o material perde cerca de $40 \%$ de sua massa inicial. O TGA do pó de talos de tabaco permite validar que, durante a preparação do filamento compósito não ocorreu a degradação do tabaco, uma vez que a temperatura média de extrusão foi de $180{ }^{\circ} \mathrm{C}$. Quanto ao PLA, sua perda de massa ocorre entre 315 e $385^{\circ} \mathrm{C}[45,46]$.

Em relação à temperatura de impressão para FFF, estudos realizados anteriormente sinalizam que a mesma se encontra em torno de 190 a $210{ }^{\circ} \mathrm{C}$. LIU et al. [47], ao desenvolverem filamento compósito para FFF de bagaço de cana de açúcar e PLA utilizaram a temperatura de $200{ }^{\circ} \mathrm{C}$ para impressão de corpos de prova. TAO et al. [32] realizaram impressões de filamento elaborado com farinha de madeira e PLA a uma temperatura de $210^{\circ} \mathrm{C}$. Impressões de corpos de prova de filamento de PLA e fibra de linho foram realizadas a $195{ }^{\circ} \mathrm{C}$ [48]. Filamentos de cortiça e PLA foram impressos a $190{ }^{\circ} \mathrm{C}$ [34]. Por fim, a impressão a partir de filamentos elaborados com lignina e PLA foram realizadas a $205{ }^{\circ} \mathrm{C}$ [49].

\section{CONCLUSÕES}

Foi desenvolvido um filamento biocompósito, constituído por um polímero biodegradável (PLA) e pó de talos de tabaco por meio de extrusora do tipo monorrosca. Verifica-se que, mediante o ajuste correto da temperatura, houve extrusão da mistura na proporção avaliada, ocorrendo sem maiores dificuldades.

A análise dos espectros de FTIR dos picos característicos do material vegetal, polimérico (PLA) e do compósito, sugere que o compósito apresenta interação mecânica entre os materiais. Essa afirmação evidencia-se com a análise das imagens de MEV, que apresentam baixa adesão interfacial entre os materiais em estudo.

Em relação a análise térmica, constata-se que a temperatura de extrusão $\left(180{ }^{\circ} \mathrm{C}\right)$ foi considerada suficiente para o processamento do compósito. Tendo em vista a análise de TGA, embora o material orgânico comece a perder água a partir dos $40{ }^{\circ} \mathrm{C}$, sua maior perda de massa concentra-se entre 300 e $600{ }^{\circ} \mathrm{C}$. Já a degradação do PLA inicia em torno de $280^{\circ} \mathrm{C}$. De acordo com o DSC, a degradação do filamento compósito inicia a uma temperatura mais baixa em comparação ao filamento de PLA virgem. Mesmo assim, essa temperatura é superior à necessária para o processo de extrusão realizado e para o processamento da tecnologia FFF. Ainda, a $\mathrm{T}_{\mathrm{m}}$ do filamento compósito, ocorre em torno de $176{ }^{\circ} \mathrm{C}$. Sendo assim, a análise térmica do filamento compósito é considerada suficiente para impressão por FFF. Conforme mencionado anteriormente, estudos realizados evidenciam que a mesma se encontra entre 190 e $210^{\circ} \mathrm{C}$. O DSC também revelou importante informação a respeito do grau de cristalinidade de ambos materiais. A adição do pó de talos de tabaco provo- 
cou um aumento no grau de cristalinidade, indicando que as partículas micronizadas desse material vegetal atuaram como agentes de nucleação para a cristalização.

A análise morfológica mostrou que a mistura de pó de talos de tabaco modifica a estrutura do PLA. As partículas de tabaco bem como as cargas do PLA estão distribuídas de modo aleatório no filamento compósito. Observa-se ainda a presença de aglomerados de partículas no centro, com interface evidente entre os aglomerados e o filamento. A utilização de agente compatibilizante pode auxiliar na melhora da compatibilidade interfacial entre a carga e a matriz hidrofóbica de PLA [36]. Durante o processo de extrusão não foram usados agentes que favorecessem a ligação. Assim, a continuidade da investigação deverá avaliar o comportamento do compósito na presença desses agentes.

Para verificar o potencial do compósito para elaboração de filamento para FFF, faz-se necessário aprofundar a pesquisa experimental, aumentando o percentual de pó de talos de tabaco e incluindo a realização de ensaios mecânicos. Como se trata de um estudo exploratório, a próxima etapa da pesquisa está voltada para investigação de diferentes composições de polímeros usados em AM como PLA e ABS e de outros métodos de extrusão e preparação de compósitos. Sugere-se, também, um estudo a respeito da adição de plastificantes ou agentes compatibilizantes, a fim de melhorar a ligação entre a interface do PLA e as partículas de tabaco.

\section{AGRADECIMENTOS}

Essa investigação contou com o apoio financeiro da Capes - Coordenação de Aperfeiçoamento de Pessoal de Nível Superior e do CNPq - Conselho Nacional de Desenvolvimento Científico e Tecnológico. Foi também desenvolvida no âmbito do projeto CICECO - Instituto de Materiais de Aveiro, UIDB/50011/2020 \& UIDP/50011/2020, financiado por fundos nacionais através da Fundação para a Ciência e Tecnologia/MCTES. Nossos agradecimentos à Faculdade da Arquitetura da UFRGS (FA/UFRGS), ao Laboratório de Materiais Cerâmicos (LACER/UFRGS), ao Laboratório de Design e Seleção de Materiais (LDSM) da Escola de Engenharia/UFRGS, à Escola Superior Aveiro Norte (ESAN) e ao Centro de Investigação de Materiais Cerâmicos e Compósitos (CICECO), ambos da Universidade de Aveiro (UA) pelo apoio técnico.

\section{BIBLIOGRAFIA}

[1] VAN WIJK, A., VAN WIJK, I., 3D printing with biomaterials: towards a sustainable and circular economy, 1 ed., Amsterdam, IOS Press, 2015.

[2] DAI, L., CHENG, T., DUAN, C., et al., "3D printing using plant-derived cellulose and its derivatives: A review", Carbohydrate Polymers, v. 203, pp.71-86, Set. 2018.

[3] MAZZANTI, V., MALAGUTTI, F., MOLLICA, F., "FDM 3D printing of polymers containing natural fillers: A review of their mechanical properties", Polymers, v. 180, pp. 51-59, Jun. 2019.

[4] BÄHR, F., WESTKÄMPER, E., "Correlations between influencing parameters and quality properties of components produced by fused deposition modeling", Procedia CIRP, v. 72, pp. 1214-1219, Mai. 2018.

[5] BALletTI, C., BALARIN, M., GUERRA, F., "3D Printing: state of art and future perspectives", Journal of Culture Heritage, v. 26, pp. 172-182, Fev. 2017.

[6] YANG, C., LIU, T., TIAN, X., et al., "3D printing for continuous fiber reinforced thermoplastic composites: mechanism and performance", Rapid Prototyping Journal, v. 23, n. 1, pp. 209-215, Jan. 2017.

[7] ALAFAGHANI, A., QATTAWIA, A., ALRAWIA, B., et al., "Experimental optimization of fused deposition modelling processing parameters: a design-for-manufacturing approach", Procedia Manufacturing, v. 10, pp. 791-803, 2017.

[8] STOOF, D., PICKERING, K., ZHANG, Y., "Fused deposition modelling of natural fibre/Polylactic acid composites", Journal of Composites Science, v. 1, n. 2, Ago. 2017.

[9] AHN, S.H., MONTERO, M., ODELL, D., et al., "Anisotropic material properties of fused deposition modeling ABS", Rapid Prototyping Journal, v. 8, n. 4, pp. 248-257, 2002.

[10] BANJANIN, B., VLADIC, G, PÁL, M., "Consistency analysis of mechanical properties of elements produced by FDM additive manufacturing technology", Revista Matéria, v. 23, n.04, Jan. 2018.

[11] THOMAS, D.S., GILBERT, S.W., Costs and cost effectiveness of additive manufacturing: A literature review and discussion, NIST 1176, National Institute od Standards and Technology. EUA, 2015.

[12] CABREIRA, V., SANTANA, R.M.C., "Effect of infill pattern in Fused Filament Fabrication (FFF) 3D Printing on materials performance", Revista Matéria, v. 25, n.03, Mar. 2020.

[13] HAMAD, K., KASEEM, M., DERI, F., "Melt Rheology of Poly(Lactic Acid)/Low Density Polyeth- 
ylene Polymer Blends", Advances in Chemical, Engineering and Science, v.1, n.4, pp.208-214, Out. 2011.

[14] FARAH, S., ANDERSON, D.G., LANGER, R., "Physical and mechanical properties of PLA, their functions in widespread applications: a comprehensive review", Advanced Drug Delivery Reviews, v. 107, pp. 367-392, Jun. 2016.

[15] SANTANA, L., ALVES, J.L., Estudo comparativo entre PETG e PLA para impressão 3D através de caracterização térmica, química e mecânica, Revista Matéria, v. 23, n. 4, Dez. 2018.

[16] MCALISTER, C., WOOD, J., "The potential of 3D printing to reduce the environmental impacts of production", In: ECEE Industrial Summer Proceedings, pp. 213-221, 2014.

[17] GUTIÉRREZ, M.C., Biocompósitos de acetato de celulose e fibras curtas de Carauá, Tese de D.Sc., Universidade Estadual de Campinas, Campinas, SP, Brasil, 2011.

[18] RAMÍREZ, M.G.L., Desenvolvimento de biocompósitos de amido termoplástico reforçados com fibra de coco verde, Tese de D.Sc., Universidade Federal do Paraná, Curitiba, PR, Brasil, 2011.

[19] JOHN, M.J., THOMAS, S., "Biofibres and biocomposites", Carbohydrate Polymers, v.71, n. 3, pp. 343364, Jun. 2007.

[20] SILVA, R.V., AQUINO, E.M.F., "Carauá fiber: a new alternative to polymeric composites", Journal Reinforced Plastic and Composites, v. 27, n. 1, pp. 103-112, Jan. 2008.

[21] ALMEIDA, N.M.B. de., Estudo estrutural de compósitos de matriz polimérica reforçados com fibras de juta, Tese de M.Sc., Universidade do Porto, Porto, Portugal, 2012.

[22] SATYANARAYANA, K.G., "Biodegradable polymer composites based on Brazilian lignocellulosic", Revista Matéria, v.15, n. 2, pp. 088-095, Jun. 2010.

[23] SINDITABACO, http://www.sinditabaco.com.br. Acessado em novembro de 2019.

[24] AFUBRA, https://afubra.com.br. Acessado em novembro de 2019.

[25] WANG, Y-J., BI, Y-Y., GAO, C-Y., "The assessment and utilization of straw resources in China", Chinese Academy of Agricultural Sciences, v.9, n.12, pp. 1807-1815, Dez. 2010.

[26] QIN, Z., SUN, M., LUO, X., et al., "Life-cycle assessment of tobacco stalk utilization", Bioresource Technology, v. 265, p. 119-127, Out. 2018.

[27] ACDA, M.N., CABANGON, R.J., "Termite resistance and physico-mechanical properties of particleboard using waste tobacco stalk and wood particles", International Biodeterioration \& Biodegradation Journal, v. 85, pp. 354-358, Nov. 2013.

[28] SHAKHES, J., MARANDI, M.A.B., ZEINALY, F., et al., "Tobacco residuals as promising lignocellulosic materials for the pulp and paper industry", Bioresources, v. 6, n. 4, pp. 4481-4493, 2011.

[29] PESEVSKI, M.D., LLIEV, B., ZIVKOVIC, D., et al., "Possibilities for utilization of tobacco stems for production of energetic briquettes", Journal of Agricultural Sciences, v.55, n.1, pp.45-54, Jan. 2010.

[30] SILVEIRA, R. L. L. da., HERMANN, E., "As cidades e a urbanização do Vale do Rio Pardo", In: Vogt, P. O., Silveira, R.L.L da. (org), Vale do Rio Pardo: (re)conhecendo a região, 1 ed., capítulo 4, Santa Cruz do Sul, Brasil, Edunisc, 2001.

[31] SILVA, S.P.M., ANTUNES, T., COSTA, M.E.V., et al., "Cork-like filaments for Additive Manufacturing", Additive Manufacturing, v. 34, Abr. 2020.

[32] TAO, Y., WANG, H., LI, P., et al., "Development and application of wood flour-filled polylactic acid composite filament for 3D Printing", Materials, v. 10, n. 4, pp. 339-345, Mar. 2017.

[33] WAHAB, M.S., WAGIMAN, A., IBRAHIM, M., "Development of wood-based composites material for 3D printing process", Applied Mechanics and Materials, v. 315, pp. 987-991, Abr. 2013.

[34] ANTUNES, T.S.J., Agentes compatibilizantes em formulações de biopolímeros com cortiça para impressão 3D, Tese de M.Sc, Universidade de Aveiro, Aveiro, Portugal, 2018.

[35] TRAN, T.N., BAYER, I.S., HEREDIA-GUERRERO, et al., "Cocoa shell waste biofilaments for 3D printing applications", Macromolecular Materials and Engineering, v. 302, n. 11, pp. 1-10, Nov. 2017.

[36] TSOU, C-Y., WU, C-L., TSOU, C-H., et al., "Biodegradable composition of poly(lactic acid) from renewable wood flour", Polymer Science Series B, v. 57, n. 5, pp. 473-480, Jul. 2015.

[37] BECKER, D., Blendas PP/PU: Estudo do efeito do agente compatibilizante e reciclagem de resíduos de $P U$, Tese de M.Sc, Universidade Federal de Santa Catarina, Florianópolis, SC, Brasil, 2002.

[38] MÉLO, T.J.A., CARVALHO, L.H., CALUMBY, R.B., et al., "Propriedades mecânicas e morfologia de uma blenda polimérica de PP/HIPS compatibilizada com SEBS", Polímeros: Ciência e Tecnologia, v. 10, n. 
2, pp. 82-89, 2000.

[39] LIDE, D.R., CRC Handbook of Chemistry and Physics, Internet Version 2005, <http://www.hbcpnetbase.com>, CRC Press, Boca Raton, FL, 2005.

[40] PAVIA, D. L., LAMPAM, G. M., KRIZ, G. S., et al., Introdução a Espectroscopia, 5 ed., Boston, Cengage, 2016.

[41] CHIENG, B.W., YUNUS, W.M.Z.W., IBRAHIM, N.A., et al., "Effects of graphene nanopletelets on Poly (lactic acid)/Poly (ethylene glycol) polymer nanocomposites", Polymers, v. 6, n. 1, pp. 93-104, Dez. 2013.

[42] MOFOKENG, J.P., TÁBI, T., LUYT, A. S., et al., "Comparison of injection moulded, natural fibre reinforced composites with PP and PLA as matrices", Journal of Thermoplastic Composite Materials, v. 25, n. 8, pp. 927-948, Dez. 2012.

[43] AURAS, R.A., HARTE, B., SELKEAND, S., et al., "Mechanical, physical and barrier properties of Poly(lactide) films", Journal of Plastic Film \& Sheeting, v. 19, n. 2, Abr. 2003.

[44] FONSECA, A.S., Caracterização tecnológica das fibras do estirpe de Desmoncus polyacanthos Mt., Tese de M.Sc, Universidade Federal de Lavras, Lavras, MG, Brasil, 2012.

[45] HARMAEN, A.S., KHALINA, A., AZOWA, I., et al., "Thermal and biodegradation properties of Poly(lactid acid)/fertilizer/oil palm fiber blends biocomposites", Polymer Composites, Mar. 2015.

[46] BACKES, E.H., PIRES, L.N., COSTA, L.C., et al., "Analysis of the degradation during melt processing of PLA/Biosilicate ${ }^{\circledR}$ composites", Journal of Composites Science, v. 3, n. 52, Mai. 2019.

[47] LIU, H., HE, H., PENG, X., et al., "Three-dimensional printing of poly(lactic acid) bio-based composites with sugarcane bagasse fiber: Effect of printing orientation on tensile performance", Polymers for Advanced Technologies, v. 30, n. 4, Jan. 2019.

[48] LE DUIGOU, A., BARBÉ, A., GUILLOU, E., et al, "3D printing of continuous flax fibre reinforced biocomposites for structural applications", Materials and Design. v. 180, Mai. 2019.

[49] GKARTZOU, E., KOUMOULOS, E.P., CHARITIDIS, C.A., "Production and 3D printing processing of bio-based thermoplastic filament", Manufacturing Review, v.4, n.1, Jan. 2017.

[50] ISO/ASTM 52900, Additive manufacturing, general principles, terminology, 2015.

\section{ORCID}

Fernanda Rech

Sara Magalhães da Silva

Liane Roldo

José Martinho Oliveira

Fabio Pinto da Silva https://orcid.org/0000-0002-5117-3820

https://orcid.org/0000-0001-9930-9486

https://orcid.org/0000-0002-0301-8631

https://orcid.org/0000-0003-1564-5728

https://orcid.org/0000-0001-9349-5601 